\title{
Key role for gene dosage and synaptic homeostasis in autism spectrum disorders
}

\author{
Roberto Toro $^{1,2}$, Marina Konyukh ${ }^{1,2}$, Richard Delorme ${ }^{1,2,3,6}$, Claire Leblond ${ }^{1,2}$, Pauline \\ Chaste $^{1,2,3}$, Fabien Fauchereau ${ }^{1,2,4}$, Mary Coleman ${ }^{5}$, Marion Leboyer ${ }^{6,7,10}$, Christopher \\ Gillberg $^{8,9}$ and Thomas Bourgeron ${ }^{1,2,4,6^{*}}$
}

${ }^{1}$ Human Genetics and Cognitive Functions, Institut Pasteur, 75015 Paris, France

${ }^{2}$ CNRS URA 2182 “Genes, synapses and cognition”, Institut Pasteur, Paris, France

${ }^{3}$ Department of Child and Adolescent Psychiatry, Hôpital Robert Debré, Assistance Publique-

Hôpitaux de Paris, 75019 Paris, France

${ }^{4}$ University Denis Diderot Paris 7, 75005 Paris, France

${ }^{5}$ Foundation for Autism Research

${ }^{6}$ Foundation FondaMental French National Science Foundation

${ }^{7}$ INSERM U955, IMRB, Psychiatry Genetic team, Faculy of Medecine, Université Paris Est-Créteil I, Créteil, France

${ }^{8}$ Department of Child and Adolescent Psychiatry, Göteborg University, 41119 Göteborg, Sweden

${ }^{9}$ Institute of Child Health, London, United Kingdom

10, AP-HP, Henri Mondor-Albert Chenevier Hospitals, Department of Psychiatry, Creteil, F-94000, France

* Correspondence should be addressed to Thomas Bourgeron, PhD, Human Genetics and Cognitive Functions, Institut Pasteur, 25 rue du Docteur Roux, 75015 Paris, France. Tel: 33-1-40-61-32-16. Fax:

33-1-40-61-39-53. E-mail: thomasb@pasteur.fr 


\begin{abstract}
Autism spectrum disorders (ASD) are characterized by impairments in reciprocal social communication, and repetitive, stereotyped verbal and non-verbal behaviors. Genetic studies have provided a relatively large number of genes that constitute a comprehensive framework to better understand this complex and heterogeneous syndrome. Based on the most robust findings, three observations can be made: first, genetic contributions to ASD are highly heterogeneous with most likely a combination of alleles with low and high penetrance. Second, the majority of the mutations apparently affect a single allele, suggesting a key role for gene dosage in the susceptibility to ASD. Finally, the broad expression and function of the causative genes suggest that alteration of synaptic homeostasis could be a common biological process associated with ASD. Understanding the mechanisms that regulate synaptic homeostasis should shed new light on the causes of ASD and may provide means to modulate the severity of the symptoms.
\end{abstract}




\section{Introduction}

The diagnosis of autism is based on impairments in two major domains - reciprocal social communication, and repetitive, stereotyped and ritualistic verbal and non-verbal behaviors. The term "autism spectrum disorders" (ASD) is used as a shorthand to refer to any patient that meets these diagnostic criteria. But beyond this unifying definition lies an extreme degree of clinical heterogeneity, ranging from profound to moderate impairments, but always with functional disability. Indeed, autism is not a single entity, but rather a complex phenotype thought to be caused by different types of defects in common pathways, producing similar behavioral phenotypes. The prevalence of ASD overall is about $1 / 100$, but closer to $1 / 300$ for typical autism ${ }^{1}$. ASD are more common in males than females with a $4: 1$ ratio $^{2,3}$. Twin and family studies have conclusively described ASD as the most "genetic" of neuropsychiatric disorders, with concordance rates of $82-92 \%$ in monozygotic twins versus $1-10 \%$ in dizygotic twins; sibling recurrence risk is $6 \%^{2,3}$.

From 15 to $70 \%$ of children diagnosed as suffering from ASD have intellectual disabilities ${ }^{4}$, and it is now understood that, like intellectual disability, autism symptoms can be caused either by gene mutations or by chromosomal aberrations (Box 1). In approximately $10-25 \%$ of the affected individuals, autism is "syndromic", ie. occurring in a child with a known genetic or environmental toxin disorder, such as fragile $\mathrm{X}$, tuberous sclerosis, neurofibromatosis, valproic syndrome, or autism caused by brain herpes simplex infection ${ }^{2,4}$.

In the last years, various independent studies and large scale international efforts have identified a growing number of candidate genes for ASD and suggest a set of mechanisms that could underlie the ASD phenotype.

\section{Genetic variations and the modes of inheritance of ASD}

Due to the absence of classical Mendelian inheritance, ASD were first thought to be a polygenic trait involving many loci. Therefore, model free linkage studies, such as affected sib-pair analyses, were performed to identify susceptibility genes. Many genomic regions were detected, but only a restricted number of loci were replicated in independent scans (e.g., chromosome 7q31 and 17q11). To homogenize the genetic and phenotypic data and to gain higher statistical power, collaborative efforts 
were initiated, such as the autism genome project (AGP), that genotyped 1496 sib-pair families using the Affymetrix 10K single nucleotide polymorphisms (SNP) array ${ }^{5}$. Nevertheless, no genome-wide significant loci could be detected, and the signals on chromosome $7 \mathrm{q} 31$ and $17 \mathrm{q} 11$ were lost. The absence of relevant targets identified by linkage studies prompted geneticists to use an alternative method: association studies with dense SNP arrays. In theory, association studies are sensitive to allelic heterogeneity whereas linkage studies are not. Nevertheless, association studies provide major advantages compared with linkage studies. First, studies can include large sample of patients since they are not restricted to multiplex families with two or more affected children. Second, the genomic regions associated with the trait are much narrower than in linkage studies, due to loss of strong linkage disequilibrium between relatively close genomic regions. In addition, SNP arrays can be used to detect structural variants such as copy number variations $(\mathrm{CNVs})^{6}$.

By using these approaches, several genes were associated with ASD. A list of 190 genes is available at AutDB, a public, curated, web-based, database for autism research (http://www.mindspec.org/autdb.html). However, it should be noted that most of these genes remain only candidates since their association was not always confirmed by replication and/or functional validation. In Tables 1 and 2, we present a list of some of these genes, and we consider the mechanisms that might be affected. Depending on the penetrance of the mutation on the risk for ASD, we derive two main categories of genes or loci. In the first (Table 1), genes or loci appear to have a high penetrance, but are mutated in a limited number of individuals (sometime a single individual). In this category, variations are mostly composed of de novo or rare point mutations, CNVs and cytogenetically detected deletions/duplications (Box 1). The second category of genes includes the socalled susceptibility genes to ASD (Table 2). Here, the variations are mostly composed of SNPs or inherited CNVs observed in the general population and associated with low risk for ASD. Especially, in this category of genes, the association with ASD should be taken with great care, since the three largest genome-wide association studies (GWAS) performed on more than 1000 patients in each study could not detect the same genes associated with $\mathrm{ASD}^{7-9}$.

Two opposite models have been proposed to explain the inheritance of ASD. Some researchers have considered autism as a polygenic trait (i.e., a group of patients with a relatively 
homogeneous phenotype produced by multiple genes, each of low effect). Others have considered autism as a very phenotypically heterogeneous group of different disorders (autisms), thought to be caused by different genes in common pathways (in this model, eventually a single highly penetrant mutation would be sufficient to produce autism). The heterogeneous model was recently supported by both the absence of strong linkage and/orassociation loci replicated by whole genome scans and by the identification of apparently monogenic forms of autism, each time affecting a limited number of patients, $1-2 \%$ for the most replicated genes. Nevertheless, the polygenic model of autism cannot be excluded for several reasons. First, it is now well established that the clinical outcome of most of the monogenic disorders are actually modulated by additional genetic variations. Second, in polygenic traits such as blood pressure and height for example, the effect of a single common variant on the phenotype seems to be very low $(\mathrm{OR}<2)$ and requires large sample size $(>15000)$ to be detected ${ }^{10}$. In ASD, the current genome scans - the largest were performed on cohorts of less than 2000 cases might lack the statistical power necessary to detect such common alleles with low effect. Finally, the number of deleterious mutations within the human genome of one individual remains difficult to establish, but it is most likely that, in a large proportion of patients, the combination of multiple rare variants such as $\mathrm{CNVs}$, coding and regulatory variations could affect specific biological pathways and therefore increase the risk for autism.

\section{Abnormal gene dosage in ASD}

In recent years, enormous progress has been achieved in detecting rare and frequent structural variants of the human genome, such as deletions, duplications and inversions ${ }^{11}$. At least eight studies have searched for such genomic imbalances in patients with ASD ${ }^{5,12-19}$. Differences in patient inclusion criteria, genotyping methodologies and algorithms to detect deletions and duplications make the comparison of these results difficult. Nevertheless, it seems that there is a significant increase in rare inherited and de novo CNVs in the ASD population compared with the general population. The overall rate of de novo CNVs in ASD could range from 5-10\% compared with $1 \%$ in the general population ${ }^{15}$. Not surprisingly, the frequency of rare and de novo CNVs increases for simplex families with one affected child (7-10\%) compared with multiplex families with at least two affected children $(2-3 \%){ }^{15}$. 
The presence of dysmorphic features in the patient also increases the odds of detecting a rare or de novo $\mathrm{CNV}$ in up to $27.5 \%{ }^{12}$. Based on the current findings, the majority of the CNVs apparently affect only one copy of the gene (which can be either deleted or duplicated), suggesting that abnormal gene dosage or expression might play a key role in the susceptibility to ASD.

Several mechanisms could explain why abnormal gene dosage would increase the risk for ASD. First, deletions may reveal a mutation on the second allele (Fig. 1A), as it has been recently shown for NRXN1 and CNTNAP2, two genes associated with ASD. Patients with Pitt-Hopkins-like syndrome (severe mental retardation, autistic behavior, epilepsy, and breathing anomalies) have been reported to carry one CNV affecting either $N R X N 1$ or $C N T N A P 2$, and a deleterious point mutation affecting the same genes on the second allele ${ }^{20}$. Therefore, this apparently dominant trait was actually a recessive trait when a mutation screening of the remaining allele was performed. In theory, the second mutation could be present only in some tissues, maybe in specific parts of the brain (Fig. 1B). This situation has not been observed in ASD yet, but is well known in familial forms of cancer where both an inherited and a somatic mutation are required to develop the disease (the "two hit" or Knudson model). Gene dosage problems may also appear even if the second copy does not present a mutation, but is silenced by epigenetic hallmarks, as it has been observed to be the case in disorders affecting imprinted loci, such as Angelman or Prader-Willi syndromes ${ }^{21}$. Similarly, allelic exclusion (i.e., the expression of a single allele in one cell) could turn off the unaffected allele in all cells or in specific cell types (Fig. 1C). Allelic exclusion was well characterized for immunoglobulins and olfactory receptors and is one of the mechanisms ensuring that a single antibody or receptor is expressed per cell $^{22}$. This mechanism, first considered as rare, could be in fact more frequent than originally thought ${ }^{23}$. Especially in the brain, allelic exclusion has been observed for cell adhesion molecules such as protocadherins ${ }^{24}$. If the cadherins and protocadherins associated with $\operatorname{ASD}^{7,14,25}$ were subject to allelic exclusion, then it would be possible that turning off the expression of the unaffected copy could totally deplete the cell of the protein product.

Second, abnormal gene dosage might by itself represent a risk factor for ASD. It is known from work in yeast that genes with high sensitivity to dosage are enriched in regulatory and structural proteins ${ }^{26}$, that need to interact with precise stoichiometry. Thus, lower or higher levels of a single 
component of these complexes might disorganize the assembly of the machinery and alter its biological function. In particular, scaffolding synaptic proteins such as SHANK involved in specific protein-protein interactions are expected to be especially sensitive to dosage (Figure 1D). Finally, some mutation associated with ASD could act as gain of function. Indeed, the knock-in mice carrying the Nlgn3 R451C mutation - previously identified in one patient with autism and his brother with Asperger syndrome ${ }^{27}$ - displayed different synaptic and behavioral phenotypes compared to the complete Nlgn3 knock-out ${ }^{28}$.

\section{Abnormal level of synaptic proteins}

Several lines of evidence indicate that mutations in genes regulating various aspects of synaptogenesis and neuronal circuit formation (Fig. 2) are associated with an increased risk for ASD. Among these, several genes seem to regulate the level of proteins at the synapse. Two X-linked genes, $M e C P 2$ and FMR1, are involved in autism "secondary" to Rett and fragile X syndromes, respectively. MeCP2 (Fig 2B) is a protein that directly and/or indirectly regulates neurotrophic factors, such as Brain Derived Neurotrophic factor (BDNF), by binding to methylated DNA ${ }^{29}$. Deletions or mutations of $M E C P 2$ are associated with Rett syndrome in females, whereas duplications of $\mathrm{MeCP} 2$ are associated with mental retardation and ASD in males and psychiatric symptoms, including generalized anxiety, depression, and compulsions in females ${ }^{30}$. FMRP (Fig 2A, B) is a selective RNA-binding protein that transports mRNA into dendrites and regulates the local translation of some of these mRNAs at synapses in response to activation of metabotropic glutamate receptors (mGluRs). In the absence of FMRP, there is an excess and a dysregulation of mRNA translation leading to altered protein synthesis dependent plasticity $^{31}$.

Mutations of other genes associated with ASD seem to affect the level of synaptic proteins by dysregulating overall cellular translation ${ }^{31}$. Patients with neurofibromatosis, tuberous sclerosis, or Cowden/Lhermitte Duclos syndromes have higher risk than the general population to have ASD. These disorders are caused by dominant mutations in the tumor suppressor genes NF1, TSC1/TSC2 and PTEN (Fig. 2). These proteins act in a common pathway as negative effectors of the rapamycinsensitive mTOR-raptor complex, a major regulator of mRNA translation and cellular growth in mitotic 
cells ${ }^{31}$. The mutations observed in ASD have been predicted to enhance the mTORC1 complex, which could lead to abnormal synaptic function due to an excess of protein synthesis. Interestingly loss of $T s c 1 / T s c 2$ or Pten in mice results in neuronal hypertrophy ${ }^{32}$, and patients presenting mutations in NF1, TSC1/TSC2 and PTEN have a higher risk for macrocephaly. Further modulation of the PTEN and MTOR pathways is exerted by serotonin and the proto-ocongene cMET, two pathways that were associated with $\operatorname{ASD}^{33,34}$.

Consistent with the hypothesis of a relationship between abnormal levels of synaptic proteins in ASD, many studies have reported mutations in genes involved in synaptic protein ubiquitination, including UBE3A, PARK2, RFWD2 and FBXO40 ${ }^{13}$ (Fig. 2A). Protein degradation through ubiquitination proceeds through the ligation of ubiquitin to the protein to be degraded. This posttranslational modification directs the ubiquitinilated proteins to cellular compartments or to degradation into the proteasome. The ligation of ubiquitin is reversible and could be used to regulate specific protein levels at the synapse. In mice, many proteins of the post-synaptic density, including the mouse orthologs of the ASD-associated SHANK proteins, have been demonstrated to be targeted by ubiquitination in an activity-dependent homeostatic manner ${ }^{35}$. Ubiquitination involves activating enzymes (E1), conjugating enzymes (E2) and ligases (E3). Substrate specificity is usually provided by the E3 ligases, which typically have substrate-binding sites. UBE3A (also called E6-AP) is an E3 ligase encoded by an imprinted gene (only expressed from the maternal copy) and is responsible for Angelman syndrome ${ }^{21}$. In ASD, de novo maternal duplications of chromosome 15q11-q13 including $U B E 3 A$ have been observed in $1-3 \%$ of the patients ${ }^{21}$. It is still not clear whether $U B E 3 A$ alone contributes to the risk of ASD, since other candidate genes are also duplicated on chromosome 15q11q13, however, its role at the synapse has been recently demonstrated in mice ${ }^{36,37}$. In cultured hippocampal neurones $U b e 3 a$ is localized at the pre- and post-synaptic compartments, but also at the nucleus. Experience-driven neuronal activity induces $U b e 3 A$ transcription, and then, Ube $3 \mathrm{~A}$ regulates excitatory synapse development by controlling the degradation of Arc, a synaptic protein that promotes the internalization of the AMPA subtype of glutamate receptors ${ }^{37}$. This might have many consequences for synaptic structure, as suggested by Ube3a maternal-deficient mice, which exhibit abnormal dendritic spine development, including spine morphology, number and length ${ }^{36}$, and a 
reduced number of AMPA receptors at excitatory synapses ${ }^{37}$.

Finally, the transcription factor $M E F 2 C$ (Fig. 2B), involved in the regulation of the number of synapses appears to be a risk factor for intellectual disability ${ }^{38}$, and could therefore also be associated with ASD. Taken together, the genetic results obtained in humans and the functional studies mostly obtained in mice suggest that different independent mechanisms could alter the level of synaptic proteins; however, the actual nature of the impaired synaptic function(s), and its association with ASD phenotype remains to be characterized.

\section{Abnormal formation of neuronal circuits in ASD}

The main category of genes associated with ASD is related to the development and the function of neuronal circuits ${ }^{39}$. At the synaptic membranes, cell adhesion molecules, such as NLGNs and NRXNs (Fig. 2) are major organizers of excitatory glutamatergic and inhibitory GABAergic synapses, and contribute to the activity-dependent formation of neuronal circuits in mice ${ }^{40}$. Mutations identified in patients with ASD were found to alter the ability of NLGNs to trigger synapse formation in cultured neuronal cells ${ }^{41,42}$. The disorders associated with NLGN-NRXN mutations can greatly vary among individuals, and this appears to be the case even for subjects of the same family, carrying the same mutation Mutations of the X-linked NLGN4X have been associated with mental retardation ${ }^{43}$, typical autism ${ }^{42,}{ }^{44}$, Asperger syndrome ${ }^{27}$ and more recently with Tourette syndrome ${ }^{45}$. In one case, a $N L G N 4 X$ deletion was observed in a male with normal intelligence and apparently no autistic features ${ }^{46}$. NRXN1, by contrast, has been implicated in disorders such as schizophrenia and Pitt-Hopkins-like syndrome, but has been also found in asymptomatic carriers ${ }^{20}$.

Interestingly, NLGN and NRXN might also play a role in social interaction in other species than humans without affecting overall cognitive functions. Mutant mice carrying a R451C Nlgn3 mutation displayed an increased number of GABAergic synapses and inhibitory currents ${ }^{28}$, normal ${ }^{47}$ to reduced social interaction ${ }^{28}$ and a reduction of ultrasonic vocalization in pups ${ }^{47}$. The knockout mice for $N \operatorname{lgn} 4$ displayed reduced social interactions and ultrasonic vocalizations at the adult stage ${ }^{48}$. By contrast, mutant knockin Nlgn3 and knockout Nlgn4 displayed enhanced to normal learning compared with wild-type mice ${ }^{28,48}$. Furthermore, in the mouse model for fragile $\mathrm{X}$, an enhanced 
Nlgn1 expression improved social behavior, whereas no effect on learning and memory was observed 49. Finally, in the honeybee, sensory deprived animals had a lower level of Nlgn1 expression, but a generally increased level of the Nlgn2-5 and NrxnI expression compared with hive bees ${ }^{50}$.

The postsynaptic density plays a major role in the organization and plasticity of the synapse, and mutations affecting scaffolding proteins, such as SHANK2, SHANK3 and DLGAP2, are recurrently found in ASD ${ }^{19,51,52}$. Deletions at 22q13 and mutations of SHANK3 could be present in more than $1-2 \%$ of ASD patients (Box 1) ${ }^{51-53}$. Shank proteins are a family of three members, which are crucial components of the postsynaptic density. Together with their binding partners, they have been shown, in vitro, to regulate the size and shape of dendritic spines ${ }^{54}$. They also link glutamate receptors to the cytoskeleton and variations in genes regulating cytoskeletal dynamics were associated with mental retardation and $\mathrm{ASD}^{19,55}$.

The role of neurotransmitter transporters and receptors in the susceptibility to ASD is still unclear. Because of the abnormally high levels of serotonin in ASD patients ${ }^{33}$, the serotonin transporter SLC6A4 was extensively analyzed, and the results pointed toward dimensional rather than categorical roles for SLC6A4 in stereotypic behaviors ${ }^{56}$. For glutamate, only weak associations for GRIK2 were detected ${ }^{57}$, and a duplication of the X-linked GRIA3 receptor gene was observed in a patient presenting typical autism ${ }^{12}$. Concerning GABA, the most robust findings concern the duplication of the GABA receptor subunit gene-cluster on chromosome 15q11-13 and the observation of maternal over-transmission of a rare variant of the GABA(A) receptor beta3 subunit gene $(\mathrm{GABRB} 3)^{58}$.

Finally, proteins, related to axonal growth and synaptic identity, are now also suspected to play a role in ASD. Semaphorins are membrane or secreted proteins (Fig. 2) that influence axon outgrowth and pruning, synaptogenesis and the density and maturation of dendritic spines. SNPs located close to the semaphorin SEMA5A were associated with ASD in a large cohort ${ }^{8}$. Independently, the level of SEMA5A mRNA was found to be lower in brain-tissue and B-lymphoblastoid cell lines from patients with ASD compared with controls ${ }^{59}$. The contactin family of proteins is involved in axonal guidance as well as in the connection between axons and glial cells, and ASD patients have been found to have deletions of the contactin genes CNTN3 and CNTN4 and the contactin associated 
protein $C N T N A P 2^{25,60-63}$. In addition, inherited CNVs or SNPs have been found in other cell-adhesion proteins - cadherins $(\mathrm{CDH} 9, \mathrm{CDH} 10, \mathrm{CDH} 18)$ and protocadherins $\mathrm{PCDH} 9$ and $\mathrm{PCDH10}{ }^{7,14,25}$ which might contribute to the susceptibility to ASD by altering neuronal identity.

\section{Abnormal synaptic homeostasis in ASD}

Different homeostatic mechanisms allow neuronal cells to maintain an optimal level of neuronal activity despite global changes in the overall activity of the network ${ }^{64-66}$. Recent evidence suggests that homeostasis plays a role in the adaptation of synaptic plasticity by changing levels of activity ${ }^{35,64}$, and might be also associated with the downscaling of synaptic weights during sleep ${ }^{65}$. During development and the first years of life, activity plays an important role in the refinement of brain connections, and many results suggest that these processes are under homeostatic control at the synapse ${ }^{67}$. The genes and the mechanisms that we have surveyed in this article might disrupt synaptic homeostasis at various levels ${ }^{68}$. The synthesis and degradation of different postsynaptic density proteins has been shown to vary as a function of activity ${ }^{35}$. Mutations in ubiquitin-dependent degradation could directly interfere with this process, as would also be the case if mutations are present in scaffolding genes such as the Shank family. Synaptic homeostasis has been shown to depend on local protein synthesis, on $\mathrm{Ca}^{2+}$ concentration and on a tight regulation between the pre- and the post synaptic sides of the synaptic contact mediated by cell adhesion molecules such as NLGN and NRXN ${ }^{69}$. Finally, synaptic homeostasis is not independent from cellular homeostasis and therefore should be affected by mutations altering gene expression level as well as neuronal numbers and shape such as mutations related to the mTOR pathway.

If synaptic homeostasis is altered in ASD, environmental factors that influence this regulatory process could also modulate its severity. As reviewed elsewhere ${ }^{33,70}$, abnormal serotonin and/or melatonin levels and altered sleep or circadian rhythms might constitute risk factors for ASD ${ }^{71}$. Sleep has been proposed as an important mechanism to regulate synaptic homeostasis. During wakefulness there appears to be a global increase in the strength of excitatory synapses, scaled down during sleep to a baseline level ${ }^{65,72}$, a mechanism that can play an important role in learning and memory ${ }^{73}$. In addition to mutations of genes directly involved in synaptic processes, we have recently proposed that, 
in some cases, ASD could result from the interplay between abnormalities in synaptic and clock genes, and that restoring circadian rhythms might therefore be beneficial for the patients and their families ${ }^{70}$.

Most of the genes considered in this review are thought to be expressed throughout the brain, however, neuroimaging studies seem to converge into a stereotypical network of brain regions where differences between ASD and control populations can be detected. These two results would not need to be in contradiction, if different brain networks were differently resilient to variations in synaptic homeostasis (Fig. 3). From an evolutionary standpoint, brain networks involved in more recently acquired cognitive skills, such as language or complex social behavior, might have less compensatory mechanisms compared with more ancient biological functions that have been shaped by a much stronger selective pressure.

\section{Concluding remarks and perspectives}

It is a matter of time for geneticists to be able to obtain whole genome sequences of ASD patients. Exploring epigenetic alterations should be also more feasible in the near future, thanks to the availability of brain tissue samples and stem cells from patients. Animal models based on genetic results are now under scrutiny in many laboratories and the consequence of the mutations and their reversibility is analyzed from cell to behavior. However, more than ever we need to recognize the inherent heterogeneity of the genetic correlates of ASD. A true understanding of the relationship between genetic mutations and ASD phenotype will not be possible if we persist in considering autism as a binary value in our analyses. Advancement in the research on ASD requires the expertise from different fields, but only clinicians and psychiatrists will be able to determine what we are actually looking at (i.e. the autism phenotype or, rather, the different autism phenotypes). If to date common variants seem to be difficult to identify in heterogeneous samples of less than 1000 families, future studies should tell if increasing sample size or meta-analyses, phenotypic stratification, pathway analyses and SNP x SNP interactions can identify common variants associated with sub groups of patients with ASD. 


\section{References}

1. Fernell, E., and Gillberg, C. Autism spectrum disorder diagnoses in Stockholm preschoolers. Res Dev Disabil

2. Freitag, C.M. (2007) The genetics of autistic disorders and its clinical relevance: a review of the literature. Mol Psychiatry 12, 2-22

3. Abrahams, B.S., and Geschwind, D.H. (2008) Advances in autism genetics: on the threshold of a new neurobiology. Nat Rev Genet 9, 341-355

4. Gillberg, C., and Coleman, M. (2000) The biology of the autistic syndromes. . Oxford University Press.

5. Szatmari, P., et al. (2007) Mapping autism risk loci using genetic linkage and chromosomal rearrangements. Nat Genet 39, 319-328

6. Cook, E.H., Jr., and Scherer, S.W. (2008) Copy-number variations associated with neuropsychiatric conditions. Nature 455, 919-923

7. Wang, K., et al. (2009) Common genetic variants on 5p14.1 associate with autism spectrum disorders. Nature 459, 528-533

8. Weiss, L.A., et al. (2009) A genome-wide linkage and association scan reveals novel loci for autism. Nature 461, 802-808

9. Anney, R., et al. (2010) A genomewide scan for common risk variants nominates phospholipase D and polysialyltransferase proteins for a role in autism. submitted

10. Manolio, T.A., et al. (2009) Finding the missing heritability of complex diseases. Nature 461, 747-753

11. Conrad, D.F., et al. (2009) Origins and functional impact of copy number variation in the human genome. Nature

12. Jacquemont, M.L., et al. (2006) Array-based comparative genomic hybridisation identifies high frequency of cryptic chromosomal rearrangements in patients with syndromic autism spectrum disorders. $J$ Med Genet 43, 843-849

13. Glessner, J.T., et al. (2009) Autism genome-wide copy number variation reveals ubiquitin and neuronal genes. Nature 459, 569-573

14. Marshall, C.R., et al. (2008) Structural variation of chromosomes in autism spectrum disorder. Am J Hum Genet 82, 477-488

15. Sebat, J., et al. (2007) Strong association of de novo copy number mutations with autism. Science 316, 445-449

16. Cho, S.C., et al. (2009) Copy number variations associated with idiopathic autism identified by whole-genome microarray-based comparative genomic hybridization. Psychiatr Genet 19, 177-185

17. Christian, S.L., et al. (2008) Novel submicroscopic chromosomal abnormalities detected in autism spectrum disorder. Biol Psychiatry 63, 1111-1117

18. Bucan, M., et al. (2009) Genome-wide analyses of exonic copy number variants in a family-based study point to novel autism susceptibility genes. PLoS Genet 5, e1000536

19. Pinto, D., et al. (2010) Functional impact of global rare copy number variation in autism spectrum disorder. Nature In press

20. Zweier, C., et al. (2009) CNTNAP2 and NRXN1 are mutated in autosomal-recessive Pitt-Hopkins-like mental retardation and determine the level of a common synaptic protein in Drosophila. Am J Hum Genet 85, 655-666

21. Schanen, N.C. (2006) Epigenetics of autism spectrum disorders. Hum Mol Genet 15 Spec No 2, R138-150

22. Serizawa, S., et al. (2004) One neuron-one receptor rule in the mouse olfactory system. Trends Genet 20, 648-653

23. Chess, A. (1998) Expansion of the allelic exclusion principle? Science 279, 2067-2068

24. Esumi, S., et al. (2005) Monoallelic yet combinatorial expression of variable exons of 
the protocadherin-alpha gene cluster in single neurons. Nat Genet 37, 171-176

25. Morrow, E.M., et al. (2008) Identifying autism loci and genes by tracing recent shared ancestry. Science 321, 218-223

26. Papp, B., et al. (2003) Dosage sensitivity and the evolution of gene families in yeast. Nature 424, 194-197

27. Jamain, S., et al. (2003) Mutations of the X-linked genes encoding neuroligins NLGN3 and NLGN4 are associated with autism. Nat Genet 34, 27-29

28. Tabuchi, K., et al. (2007) A neuroligin-3 mutation implicated in autism increases inhibitory synaptic transmission in mice. Science 318, 71-76

29. Chahrour, M., et al. (2008) MeCP2, a key contributor to neurological disease, activates and represses transcription. Science 320, 1224-1229

30. Ramocki, M.B., et al. (2009) Autism and other neuropsychiatric symptoms are prevalent in individuals with MeCP2 duplication syndrome. Ann Neurol 66, 771-782

31. Kelleher, R.J., 3rd, and Bear, M.F. (2008) The autistic neuron: troubled translation? Cell 135, 401-406

32. Tavazoie, S.F., et al. (2005) Regulation of neuronal morphology and function by the tumor suppressors Tsc1 and Tsc2. Nat Neurosci 8, 1727-1734

33. Cook, E.H., and Leventhal, B.L. (1996) The serotonin system in autism. Curr Opin Pediatr 8, 348-354.

34. Campbell, D.B., et al. (2006) A genetic variant that disrupts MET transcription is associated with autism. Proc Natl Acad Sci U S A 103, 16834-16839

35. Ehlers, M.D. (2003) Activity level controls postsynaptic composition and signaling via the ubiquitin-proteasome system. Nat Neurosci 6, 231-242

36. Dindot, S.V., et al. (2008) The Angelman syndrome ubiquitin ligase localizes to the synapse and nucleus, and maternal deficiency results in abnormal dendritic spine morphology. Hum Mol Genet 17, 111-118

37. Greer, P.L., et al. (2010) The Angelman Syndrome Protein Ube3A Regulates Synapse Development by Ubiquitinating Arc. Cell 140, 704-716

38. Le Meur, N., et al. (2009) MEF2C haploinsufficiency caused either by microdeletion of the $5 \mathrm{q} 14.3$ region or mutation is responsible for severe mental retardation with stereotypic movements, epilepsy and/or cerebral malformations. J Med Genet

39. Bourgeron, T. (2009) A synaptic trek to autism. Curr Opin Neurobiol 19, 231-234

40. Sudhof, T.C. (2008) Neuroligins and neurexins link synaptic function to cognitive disease. Nature 455, 903-911

41. Chih, B., et al. (2004) Disorder-associated mutations lead to functional inactivation of neuroligins. Hum Mol Genet 13, 1471-1477

42. Zhang, C., et al. (2009) A neuroligin-4 missense mutation associated with autism impairs neuroligin-4 folding and endoplasmic reticulum export. J Neurosci 29, 10843-10854

43. Laumonnier, F., et al. (2004) X-linked mental retardation and autism are associated with a mutation in the NLGN4 gene, a member of the neuroligin family. Am J Hum Genet 74, $552-557$

44. Jamain, S., et al. (2003) Mutations of the X-linked genes encoding neuroligins NLGN3 and NLGN4 are associated with autism. Nature Genetics 34, 27-29

45. Lawson-Yuen, A., et al. (2008) Familial deletion within NLGN4 associated with autism and Tourette syndrome. Eur J Hum Genet 16, 614-618

46. Macarov, M., et al. (2007) Deletions of VCX-A and NLGN4: a variable phenotype including normal intellect. J Intellect Disabil Res 51, 329-333

47. Chadman, K.K., et al. (2008) Minimal aberrant behavioural phenotypes of Neuroligin3 R451C knockin mice. Autism Research 1, 147-158.

48. Jamain, S., et al. (2008) Reduced social interaction and ultrasonic communication in a 
mouse model of monogenic heritable autism. Proc Natl Acad Sci U S A 105, 1710-1715

49. Dahlhaus, R., and El-Husseini, A. (2009) Altered Neuroligin expression is involved in social deficits in a mouse model of the Fragile X Syndrome. Behav Brain Res

50. Biswas, S., et al. (2010) Sensory regulation of neuroligins and neurexin $I$ in the honeybee brain. PLoS One 5, e9133

51. Durand, C.M., et al. (2007) Mutations in the gene encoding the synaptic scaffolding protein SHANK3 are associated with autism spectrum disorders. Nat Genet 39, 25-27

52. Moessner, R., et al. (2007) Contribution of SHANK3 mutations to autism spectrum disorder. Am J Hum Genet 81, 1289-1297

53. Gauthier, J., et al. (2009) Novel de novo SHANK3 mutation in autistic patients. Am J Med Genet B Neuropsychiatr Genet 150B, 421-424

54. Roussignol, G., et al. (2005) Shank expression is sufficient to induce functional dendritic spine synapses in aspiny neurons. $J$ Neurosci $25,3560-3570$

55. Persico, A.M., and Bourgeron, T. (2006) Searching for ways out of the autism maze: genetic, epigenetic and environmental clues. Trends Neurosci 29, 349-358

56. Sutcliffe, J.S., et al. (2005) Allelic heterogeneity at the serotonin transporter locus (SLC6A4) confers susceptibility to autism and rigid-compulsive behaviors. Am J Hum Genet 77, 265-279

57. Jamain, S., et al. (2002) Linkage and association of the glutamate receptor 6 gene with autism. Molecular Psychiatry 7, 302-310

58. Delahanty, R.J., et al. (2009) Maternal transmission of a rare GABRB3 signal peptide variant is associated with autism. Mol Psychiatry

59. Melin, M., et al. (2006) Constitutional downregulation of SEMA5A expression in autism. Neuropsychobiology 54, 64-69

60. Alarcon, M., et al. (2008) Linkage, association, and gene-expression analyses identify CNTNAP2 as an autism-susceptibility gene. Am J Hum Genet 82, 150-159

61. Arking, D.E., et al. (2008) A common genetic variant in the neurexin superfamily member CNTNAP2 increases familial risk of autism. Am J Hum Genet 82, 160-164

62. Bakkaloglu, B., et al. (2008) Molecular cytogenetic analysis and resequencing of contactin associated protein-like 2 in autism spectrum disorders. Am J Hum Genet 82, 165173

63. Roohi, J., et al. (2009) Disruption of contactin 4 in three subjects with autism spectrum disorder. J Med Genet 46, 176-182

64. Turrigiano, G.G. (1999) Homeostatic plasticity in neuronal networks: the more things change, the more they stay the same. Trends Neurosci 22, 221-227

65. Tononi, G., and Cirelli, C. (2003) Sleep and synaptic homeostasis: a hypothesis. Brain Res Bull 62, 143-150

66. Macleod, G.T., and Zinsmaier, K.E. (2006) Synaptic homeostasis on the fast track. Neuron 52, 569-571

67. Turrigiano, G.G., and Nelson, S.B. (2004) Homeostatic plasticity in the developing nervous system. Nat Rev Neurosci 5, 97-107

68. Ramocki, M.B., and Zoghbi, H.Y. (2008) Failure of neuronal homeostasis results in common neuropsychiatric phenotypes. Nature 455, 912-918

69. Yu, L.M., and Goda, Y. (2009) Dendritic signalling and homeostatic adaptation. Curr Opin Neurobiol 19, 327-335

70. Bourgeron, T. (2007) The possible interplay of synaptic and clock genes in autism spectrum disorders. Cold Spring Harb Symp Quant Biol 72, 645-654

71. Melke, J., et al. (2008) Abnormal Melatonin Synthesis in Autism Spectrum Disorders. Mol Psychiatry May 15

72. Miller, G. (2009) Neuroscience. Sleeping to reset overstimulated synapses. Science 
324,22

73. Stickgold, R., and Walker, M.P. (2005) Memory consolidation and reconsolidation: what is the role of sleep? Trends Neurosci 28, 408-415

74. Somerville, M.J., et al. (2005) Severe expressive-language delay related to duplication of the Williams-Beuren locus. N Engl J Med 353, 1694-1701

75. Weiss, L.A., et al. (2008) Association between microdeletion and microduplication at 16p11.2 and autism. N Engl J Med 358, 667-675

76. Kumar, R.A., et al. (2008) Recurrent 16p11.2 microdeletions in autism. Hum Mol Genet 17, 628-638

77. McCarthy, S.E., et al. (2009) Microduplications of $16 \mathrm{p} 11.2$ are associated with schizophrenia. Nat Genet 41, 1223-1227

78. Elsea, S.H., and Girirajan, S. (2008) Smith-Magenis syndrome. Eur J Hum Genet 16, 412-421

79. Potocki, L., et al. (2007) Characterization of Potocki-Lupski syndrome $(\operatorname{dup}(17)(\mathrm{p} 11.2 \mathrm{p} 11.2))$ and delineation of a dosage-sensitive critical interval that can convey an autism phenotype. Am J Hum Genet 80, 633-649

80. Niklasson, L., et al. (2009) Autism, ADHD, mental retardation and behavior problems in 100 individuals with 22q11 deletion syndrome. Res Dev Disabil 30, 763-773

81. Cai, G., et al. (2008) Multiplex ligation-dependent probe amplification for genetic screening in autism spectrum disorders: Efficient identification of known microduplications and identification of a novel microduplication in ASMT. BMC Med Genomics 1, 50

82. Gauthier, J., et al. De novo mutations in the gene encoding the synaptic scaffolding protein SHANK3 in patients ascertained for schizophrenia. Proc Natl Acad Sci U S A 107, 7863-7868

83. Garber, K.B., et al. (2008) Fragile X syndrome. Eur J Hum Genet 16, 666-672

84. Amir, R.E., et al. (1999) Rett syndrome is caused by mutations in X-linked MECP2, encoding methyl-CpG-binding protein 2. Nature Genetics 23, 185-188.

85. Wiznitzer, M. (2004) Autism and tuberous sclerosis. J Child Neurol 19, 675-679

86. Rosser, T.L., and Packer, R.J. (2003) Neurocognitive dysfunction in children with neurofibromatosis type 1. Curr Neurol Neurosci Rep 3, 129-136

87. Butler, M.G., et al. (2005) Subset of individuals with autism spectrum disorders and extreme macrocephaly associated with germline PTEN tumour suppressor gene mutations. $J$ Med Genet 42, 318-321

88. Splawski, I., et al. (2006) CACNA1H mutations in autism spectrum disorders. $J$ Biol Chem 281, 22085-22091

89. Feng, J., et al. (2006) High frequency of neurexin 1 beta signal peptide structural variants in patients with autism. Neurosci Lett 409, 10-13

90. Fernandez, T., et al. (2008) Disruption of Contactin 4 (CNTN4) results in developmental delay and other features of 3p deletion syndrome. Am J Hum Genet 82, 1385

91. Verkerk, A.J., et al. (2003) CNTNAP2 is disrupted in a family with Gilles de la Tourette syndrome and obsessive compulsive disorder. Genomics 82, 1-9

92. Friedman, J.I., et al. (2008) CNTNAP2 gene dosage variation is associated with schizophrenia and epilepsy. Mol Psychiatry 13, 261-266

93. Castermans, D., et al. (2003) The neurobeachin gene is disrupted by a translocation in a patient with idiopathic autism. $J$ Med Genet 40, 352-356

94. Gauthier, J., et al. (2008) Novel de novo SHANK3 mutation in autistic patients. Am J Med Genet B Neuropsychiatr Genet

95. Jamain, S., et al. (2003) Mutations of the X-linked genes encoding neuroligins NLGN3 and NLGN4 are associated with autism. Nat Genet 34, 27-29

96. Piton, A., et al. (2008) Mutations in the calcium-related gene IL1RAPL1 are 
associated with autism. Hum Mol Genet 17, 3965-3974

97. Melke, J., et al. (2008) Abnormal melatonin synthesis in autism spectrum disorders. Mol Psychiatry 13, 90-98

98. Toma, C., et al. (2007) Is ASMT a susceptibility gene for autism spectrum disorders? A replication study in European populations. Mol Psychiatry 12, 977-979

99. Williams, J.M., et al. (2009) A 1q42 deletion involving DISC1, DISC2, and TSNAX in an autism spectrum disorder. Am J Med Genet A 149A, 1758-1762

100. Petek, E., et al. (2007) Molecular and genomic studies of IMMP2L and mutation screening in autism and Tourette syndrome. Mol Genet Genomics 277, 71-81

101. Turunen, J.A., et al. (2008) Mitochondrial aspartate/glutamate carrier SLC25A12 gene is associated with autism. Autism Res 1, 189-192

102. Ramoz, N., et al. (2004) Linkage and association of the mitochondrial aspartate/glutamate carrier SLC25A12 gene with autism. Am J Psychiatry 161, 662-669

103. Skaar, D.A., et al. (2005) Analysis of the RELN gene as a genetic risk factor for autism. Mol Psychiatry 10, 563-571

104. Bonora, E., et al. (2005) Mutation screening and association analysis of six candidate genes for autism on chromosome 7q. Eur J Hum Genet 13, 198-207

105. Benayed, R., et al. (2005) Support for the homeobox transcription factor gene ENGRAILED 2 as an autism spectrum disorder susceptibility locus. Am J Hum Genet 77, 851-868

106. Barnby, G., et al. (2005) Candidate-gene screening and association analysis at the autism-susceptibility locus on chromosome 16p: evidence of association at GRIN2A and ABAT. Am J Hum Genet 76, 950-966

107. Devlin, B., et al. (2005) Autism and the serotonin transporter: the long and short of it. Mol Psychiatry 10, 1110-1116

108. Weiss, L.A., et al. (2006) Variation in ITGB3 is associated with whole-blood serotonin level and autism susceptibility. Eur J Hum Genet 14, 923-931

109. Kilpinen, H., et al. (2009) Linkage and linkage disequilibrium scan for autism loci in an extended pedigree from Finland. Hum Mol Genet 18, 2912-2921 


\section{Box 1. Matched syndromes of reciprocal imprinting or deletions and duplications}

A series of matched syndromes have been found which have aberrations in identical or very similar genomic regions. These syndromes usually contain subgroups with autistic features, but each matched pair of syndromes might have different symptoms in other clinical categories.

On chromosome 7q11, a microdeletion causes the Williams-Beuren syndrome, which is the opposite of autism: if anything, they are overly friendly and have strengths in language, face processing, and sociability. Strikingly, the same genomic region was found duplicated in several patients with severely impaired articulation and expressive speech abilities ${ }^{74}$.

On chromosome 15q11-13, maternal deletions cause Angelman syndrome (40-80\% autism), which has mental retardation, handflapping and mild spasticity or ataxia ${ }^{13,21}$. Paternal deletions or maternal UPD cause the Prader-Willi syndrome of hypotonia, obesity, short stature with small hands and feet and hypogonadotropic hypogonadism. Regarding duplications, maternal duplications are a relatively common cause of ASD with autistic features found in $40 \%$ of patients with the duplications ${ }^{21}$.

On chromosome $16 \mathrm{p} 11.2$, deletions and duplications of $\sim 600 \mathrm{~kb}$ are inherited or de novo and were identified in $0.5-1 \%$ of the patients with $\mathrm{ASD}^{14,75,76}$. The duplication of the same region is also associated with schizophrenia ${ }^{14,75-77}$.

On chromosome 17p11.2, deletions cause Smith-Magenis syndrome characterized by infant hypotonia, short stature, a dysmorphic face, mental retardation, congenital cardiac defects, inverted melatonin cycles and often sleep disorders and self-abusive behaviors ${ }^{78}$. Duplication of the same genomic region causes the Potocki-Lupski syndrome where about two-thirds of the children meet ASD criteria $^{79}$.

On chromosome 22q11.2, deletions are mostly de novo and encompass 1.5 to $3 \mathrm{Mb}$ of genomic DNA including 24-30 genes. The patients present with the DiGeorge 1 syndrome, where 50\% meet autism criteria ${ }^{80}$. Other individuals might have learning disabilities or schizophrenia ${ }^{80}$. Reciprocal duplications are mostly inherited from healthy parents and these children also have a higher risk for ASD with hypotonia and both mental and growth retardation ${ }^{81}$. 
On chromosome 22q13.3, the microdeletion syndrome is characterized by normal to accelerated growth combined with neonatal hypotonia, minor dysmorphic features, absent to severely delayed speech and global developmental delay ${ }^{51}$. Up to $44 \%$ of these children meet autistic criteria and a duplication of the same region, including the SHANK3 gene, was observed in a boy with Asperger syndrome who learned language preciously, in a girl with ADHD and in several patients with schizophrenia ${ }^{51,52,82}$. 


\section{Figure legends}

Figure 1. Figure 1. Possible consequences of abnormal gene dosage. Abnormal gene dosage seems to play a key role in the susceptibility to ASD. Several mechanisms can explain how abnormal gene dosage can alter brain development. In panel A, a gene has two alleles, A1 and A2 (in yellow and blue respectively, left column). The deletion of allele A1 could completely devoid the cell of the gene product if the other allele A2 is mutated (middle column) as it has been shown for patients with NRXN1 and CNTNAP2 deletions; or silenced by epigenetic hallmarks as in Angelman or Prader-Willi syndromes (right column). The second mutation could devoid the brain from the gene product only in specific regions (panel B) if, as in the "two-hits" or Knudson model, the first allele presented a germinal mutation while the mutation in the second allele (somatic mutation) occurred later during development. Another mechanism involves allelic exclusion, which has been already observed for cell adhesion molecules (panel C). For example, if the protocadherins involved in ASD were subject to allelic exclusion, brain cells would produce either allele A1 or A2 in the normal case (left side of panel C). If allele A1 was deleted, then no protein would be produced in the cells where A2 is silent, and only A2 would be present in the brain, produced by the remaining cells. Finally, an imbalance of gene product due to a deletion or a duplication could lead to disorganized protein complexes. Scaffold proteins (such as the SHANK family) at the post-synaptic density assemble with other proteins in a precise stoichiometry (panel D, left). Both the absence (middle) or excess (right) of protein could affect this equilibrium.

Figure 2. Biological processes involved in ASD. The genes associated with ASD appear to participate into three main biological processes. First (panel A), at the synapse cell adhesion proteins such as cadherins $(\mathrm{CDH})$, protocadherins $(\mathrm{PCDH})$, neuroligins (NLGN) and neurexins (NRXN) are involved in synaptic recognition and assembly. Within the postsynaptic density, scaffold proteins such as SHANK3 and DLGAP2 assemble the synaptic components and provide a link between membrane proteins and the actin skeleton. FMRP transports mRNA at the dendrites and regulates local translation of synaptic proteins. In the cytoplasm, the mTOR pathway regulates translation and is influenced by proteins such as PTEN, NF1, TSC1/TS2 and c-MET. The E3 ligase UBE3A is involved in the 
targeting of synaptic proteins to the proteasome. Receptors for glutamate (GLUR) and GABA (GABAR) are playing a central role in producing excitatory and inhibitory currents, respectively. IMPP2L is a peptidase within the inner membrane of the mitochondria. Second (panel B), in the nucleus the methyl binding protein MECP2 and transcription factors such as MEF2C regulate the expression of neuronal genes involved in the formation of neuronal circuits and synaptic functions. The FMRP protein transports and regulates the translation of mRNA at the synapse. Finally (panel C), at the nodes of Ranvier proteins such as CNTN and CNTNAP2 organize the tight junctions between the axon and the myelinating glia. At the membrane or in the intercellular space, cell adhesion molecules and secreted proteins such as NRCAM or SEMA5A act as guidance cues for axonal outgrowth.

Figure 3. Different resilience of brain networks to gene dosage. Depending on their different resilience, the functional effects of abnormal gene-dosage could seem localized, even if the genetic abnormalities are widespread. Brain networks involved in evolutionary older biological processes might have developed more compensatory mechanisms than those supporting more recent cognitive functions. In the illustration we distinguish four possibilities, networks insensitive to gene-dosage (dark blue), networks only sensitive to duplications (light blue) or deletions (orange), and finally, those unable to compensate for gene-dosage abnormalities (red). Nodes represent brain regions, and edges between nodes represent their functional link. Wavy edges represent sub-optimal functional links.

\section{Databases used in this review}

DECIPHER v4.3 https://decipher.sanger.ac.uk/application/

Autism Genetic Database (AGD) http://wren.bcf.ku.edu/

Autism CNV Database http://projects.tcag.ca/autism_500k/

AutDB http://www.mindspec.org/autdb.html

BioGPS http://biogps.gnf.org/\#goto=welcome

UCSC Genome browser http://genome.ucsc.edu 


\section{Acknowledgments}

This work was supported by the Institut Pasteur, Université Denis Diderot Paris 7, INSERM, CNRS, Fondation Orange, Fondation de FranceFondation FondaMentale. 
Table 1. Genes associated with high risk for ASD

\begin{tabular}{|c|c|c|c|c|c|c|}
\hline Gene & Chromosome & Function & Evidence & Inheritance & Diagnosis & Reference \\
\hline FMR1 & $\mathrm{Xq} 27$ & Synaptic translation & Mutations & de novo (permutations) & ASD, Fragile X syndrome & 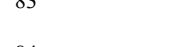 \\
\hline MECP2 & $\mathrm{Xq} 26$ & Chromatin remodeling & $\mathrm{CNV}$, mutations & de novo (rarely inherited) & ASD, Rett syndrome & 84 \\
\hline$T S C l$ & $9 \mathrm{q} 34.13$ & mTOR / PI3K pathway & $\mathrm{CNV}$, mutations & de novo, inherited & ASD, Tuberous sclerosis & \\
\hline$N F 1$ & $17 \mathrm{q} 11.2$ & mTOR / PI3K pathway & $\mathrm{CNV}$, mutation & de novo, inherited & ASD, Neurofibromatosis & \\
\hline PTEN & $10 \mathrm{q} 23.31$ & mTOR / PI3K pathway & $\mathrm{CNV}$, mutations & de novo, inherited & ASD, Cowden syndrome & 81 \\
\hline CACNAIC & $12 \mathrm{p} 13.33$ & Calcium channel & Mutation & de novo & ASD, Timothy syndrome & $8 \gamma$ \\
\hline$R F W D 2$ & 1q25.1-q25.2 & Ubiquitination & $\mathrm{CNV}$ & de novo, inherited & ASD & 13 \\
\hline$N R X N 1$ & $2 \mathrm{p} 16.3$ & Synaptic CAM & $\mathrm{CNV}$, mutations, SNP & de novo, inherited & ASD, SCZ & \\
\hline CNTN4 & $3 \mathrm{p} 26.3$ & Synaptic CAM & $\mathrm{CNV}$ & Inherited & ASD, MR & $\begin{array}{l}13,25,63 \\
38\end{array}$ \\
\hline$M E F 2 C$ & $5 q 14.3$ & Transcription factor & $\mathrm{CNV}$, mutations & de novo & MR, Seizures & 38 \\
\hline SYNGAP1 & $6 \mathrm{p} 21.3$ & Synaptic Ras GAP & $\mathrm{CNV}$ & de novo & ASD, MR & - \\
\hline CNTNAP2 & $7 \mathrm{q} 35-7 \mathrm{q} 36.1$ & Synaptic CAM & $\mathrm{CNV}$, rare variants * & Inherited & ASD, MR, SCZ, TS, & $20,00-02,91,92$ \\
\hline$N B E A$ & $13 \mathrm{q} 13.2$ & Synaptic protein & Translocation & de novo & ASD & 93 \\
\hline UBE3A & $15 \mathrm{q} 11-\mathrm{q} 13$ & Ubiquitination & $\mathrm{CNV}$ & de novo, inherited & ASD & 10 \\
\hline SHANK3 (del 22q13) & $22 q 13$ & Synaptic scaffold & $\mathrm{CNV}$, mutations & de novo, inherited & ASD, MR, SCZ & 51,5294 \\
\hline NLGN3 & $\mathrm{Xq} 13.1$ & Synaptic CAM & Mutation & Inherited & ASD & 95 \\
\hline IL1RAPLI & $\mathrm{Xp} 21.3-\mathrm{p} 21.2$ & Synaptic receptor & $\mathrm{CNV}$, mutations & de novo, inherited & ASD, MR & 96 \\
\hline NLGN4 & Xp22 & Synaptic CAM & $\mathrm{CNV}$, mutations & de novo, inherited & ASD, MR, TS & 95 \\
\hline PTCHD1 & Xp22.11 & Hedgehog receptor activity & $\mathrm{CNV}$ & Inherited & ASD & 14 \\
\hline GRIA3 & Xp25 & Synaptic receptor & $\mathrm{CNV}$ & Inherited & ASD & \\
\hline
\end{tabular}

ASD Autism Spectrum Disorder ; SCZ Schizophrenia; MR Mental Retardation; ADHD Attention-Deficit Hyperactivity Disorder ; MDC1D congenital muscular dystrophy ;

BP Bipolar, TS Tourette syndrome; * in contrast to mutations, the functional role of the rare variants was not confirmed 
Table 2. Proposed susceptibility genes for ASD

\begin{tabular}{|c|c|c|c|c|c|}
\hline Gene & Chromosome & Function & Evidence & Diagnosis & References \\
\hline$A S M T$ & PAR1 & Melatonin pathway & Inherited CNV, SNPs, mutations & ASD & $81,97,98$ \\
\hline DISC1/DISC2 & $1 q 42.2$ & Axonal growth & Inherited CNV & ASD, SCZ & 99 \\
\hline TSNAX & $1 \mathrm{q} 42.2$ & Cell differentiation & Inherited CNV & ASD, SCZ & 99 \\
\hline$D P P 10$ & $2 q 14.1$ & Dipeptidyl-peptidase activity & Inherited CNV & ASD & 14 \\
\hline CNTN3 & $3 p 12.3$ & Synaptic CAM & Inherited CNV & ASD & 25 \\
\hline FBXO40 & $3 q 13.3$ & Unknown function & Inherited CNV P $=3.3 \times 10^{-3}$ & ASD & 13 \\
\hline SLC9A9 & $3 q 24$ & Transporter & Inherited $\mathrm{CNV}$, mutations & ASD, ADHD, MR & 25 \\
\hline PCDH10 & $4 \mathrm{q} 28$ & Synaptic CAM & Inherited CNV & ASD & 25 \\
\hline PARK2 & $6 \mathrm{q} 26$ & Ubiquitination & Inherited CNV P $=3.3 \times 10^{-3}$ & ASD, PD & 13 \\
\hline$I M M P 2 L$ & $7 \mathrm{q} 31.1$ & Mitochondrial protease & Inherited CNV & ASD, TS, ADHD & 100 \\
\hline PCDH9 & $13 q 21$ & Synaptic CAM & Inherited CNV & ASD & 14,25 \\
\hline BZRAPI & $17 \mathrm{q} 22$ & Benzodiazepine receptor binding & Inherited $\mathrm{CNV} P=2.3 \times 10^{-5}$ & ASD & 18 \\
\hline PLD5 & $1 q 43$ & Phospholipase D & SNP rs2196826 P $=1.1 \times 10^{-8}$ & ASD & 9 \\
\hline$S L C 25 A 12$ & $2 \mathrm{q} 31.1$ & Synaptic receptor & SNP rs2056202 $\mathrm{P}=1 \times 10^{-3}$ & ASD & 101,102 \\
\hline $\mathrm{CDH} / \mathrm{CDH} 10$ & $5 \mathrm{p} 14.2$ & Synaptic CAM & SNP rs4307059P $=3.4 \times 10^{-8}$ & ASD & 7 \\
\hline SEMASA & $5 \mathrm{p} 15.2$ & Axonal guidance & SNP rs $10513025 \mathrm{P}=2 \times 10^{-7}$ & ASD & 8 \\
\hline TAS2R1 & $5 \mathrm{p} 15.2$ & Receptor & SNP rs $10513025 \mathrm{P}=2 \times 10^{-7}$ & ASD & 8 \\
\hline GRIK2 & $6 \mathrm{q} 16.3$ & Synaptic receptor & SNP rs3213607 P $=0.02$ & ASD, SCZ, OCD, MR & 57 \\
\hline POU6F2 & $7 \mathrm{p} 14.1$ & Transcription factor & SNP rs10258862 $\mathrm{P}=4.4 \times 10^{-7}$ & ASD & 9 \\
\hline RELN & $7 \mathrm{q} 22.1$ & Axonal guidance & GGC repeat in the $5^{\prime}$ UTR $\mathrm{P}<0.05$ & $\mathrm{ASD}, \mathrm{BP}$ & 103 \\
\hline NRCAM & $7 \mathrm{q} 31.1$ & Synaptic receptor & SNP rs2300045 $\mathrm{P}=0.017$ & ASD & 104 \\
\hline$M E T$ & $7 \mathrm{q} 31.2$ & Tyrosine kinase & SNP rs $1858830 \mathrm{P}=2 \times 10^{-3}$ & ASD & 34 \\
\hline EN2 & $7 \mathrm{q} 36.3$ & Transcription factor & SNP rs $1861972 \mathrm{P}=9 \times 10^{-3}$ & ASD & 105 \\
\hline ST8SIA2 & $15 \mathrm{q} 26.1$ & $\mathrm{~N}$-glycan processing & $\mathrm{SNP} \operatorname{rs} 3784730 \mathrm{P}=4 \times 10^{-7}$ & ASD & 9 \\
\hline GRIN2A & $16 \mathrm{p} 13.2$ & Synaptic receptor & SNP rs1014531 P $=2.9 \times 10^{-7}$ & ASD, SCZ & 106 \\
\hline$A B A T$ & $16 \mathrm{p} 13.2$ & Enzyme & $\mathrm{SNP}$ rs $1731017 \mathrm{P}=1 \times 10^{-3}$ & ASD, GABA-AT Deficiency & 106 \\
\hline SLC6A4 & $17 \mathrm{q} 11.2$ & Serotonin Transporter & Meta analysis $\mathrm{P}>0.05$ & ASD, OCD & 107 \\
\hline ITGB3 & $17 \mathrm{q} 21.3$ & Cell-matrix adhesion & SNP Leu33Pro $\mathrm{P}=8.2 \times 10^{-4}$ & ASD & 108 \\
\hline TLE2 / TL6 & $19 \mathrm{p} 13$ & Wnt receptor signaling pathway & SNP rs4806893 P $=7.8 \times 10^{-5}$ & ASD, FHM2, AHC & 109 \\
\hline MACROD2 & $20 \mathrm{p} 12$ & Unknown function & SNP rs4141463 P $=2 \times 10^{-8}$ & ASD & 9 \\
\hline
\end{tabular}

ASD Autism Spectrum Disorder ; SCZ Schizophrenia; PD Parkinson Disease; TS Tourette syndrome; ADHD Attention-Deficit Hyperactivity Disorder ; MR Mental Retardation; FHM2 Familial Hemiplegic Migraine 2 ; AHC Alternating Hemiplegia of Childhood ; OCD Obsessive-Compulsive Disorder ; BP Bipolar Disorder . 


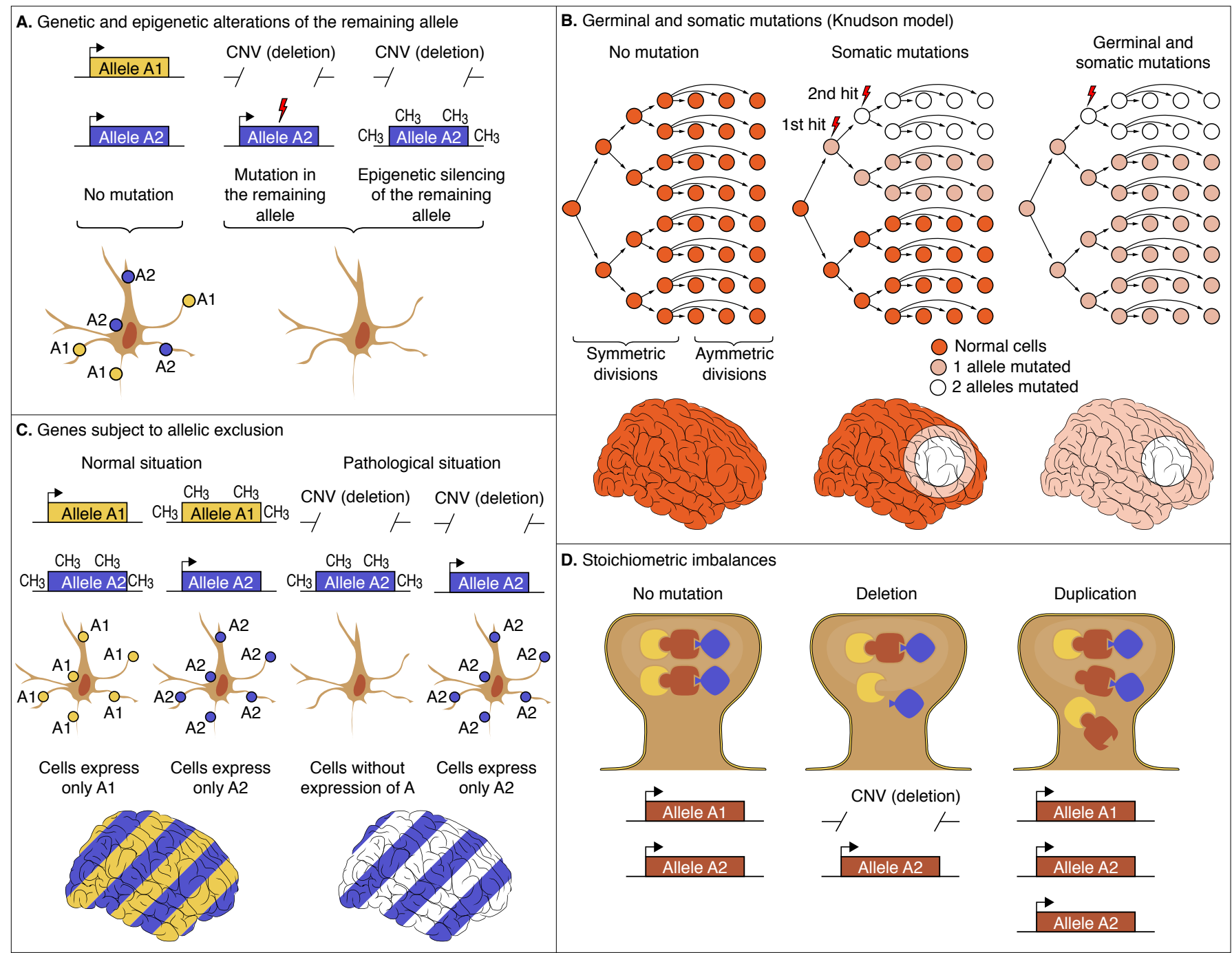



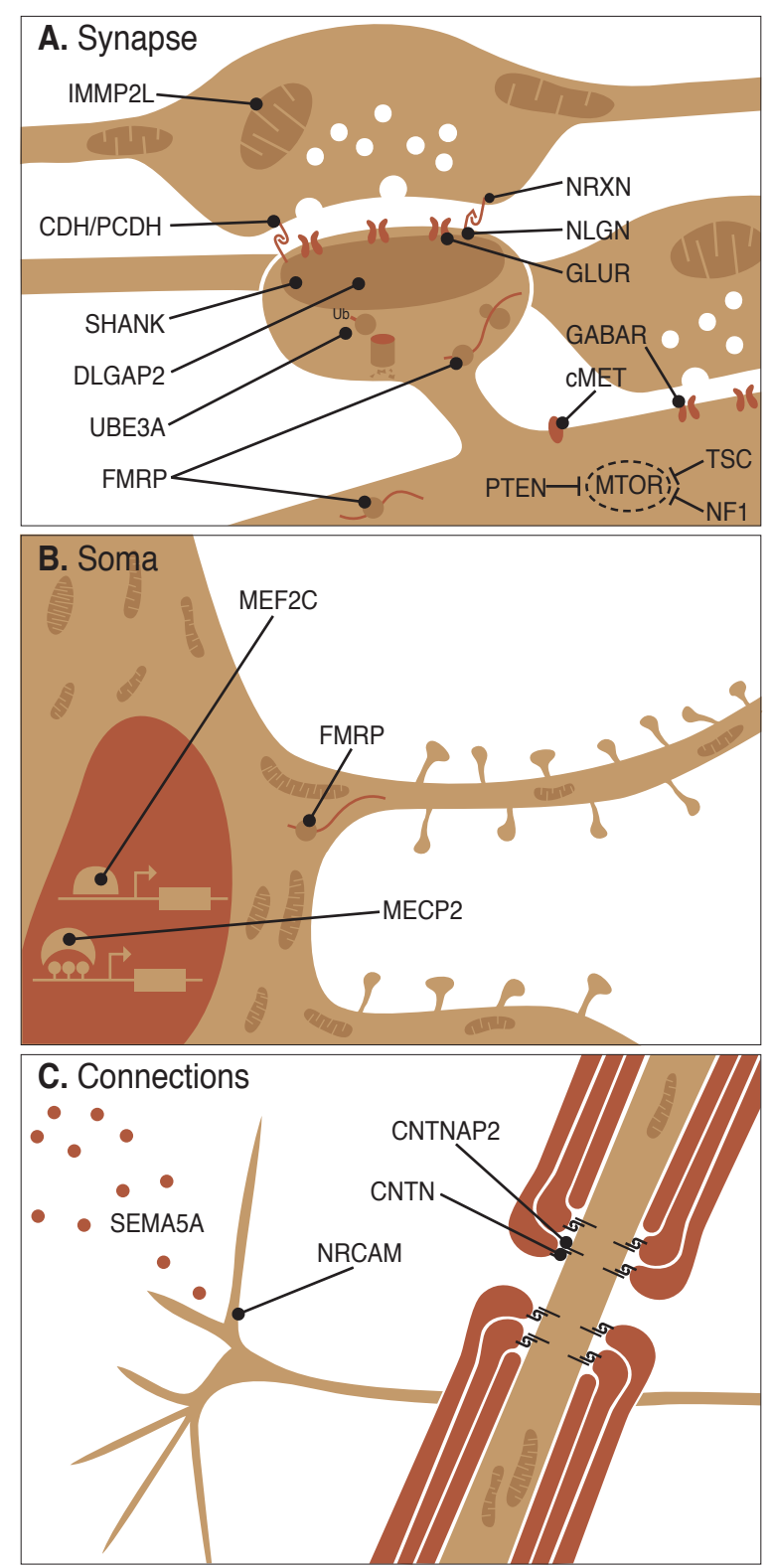


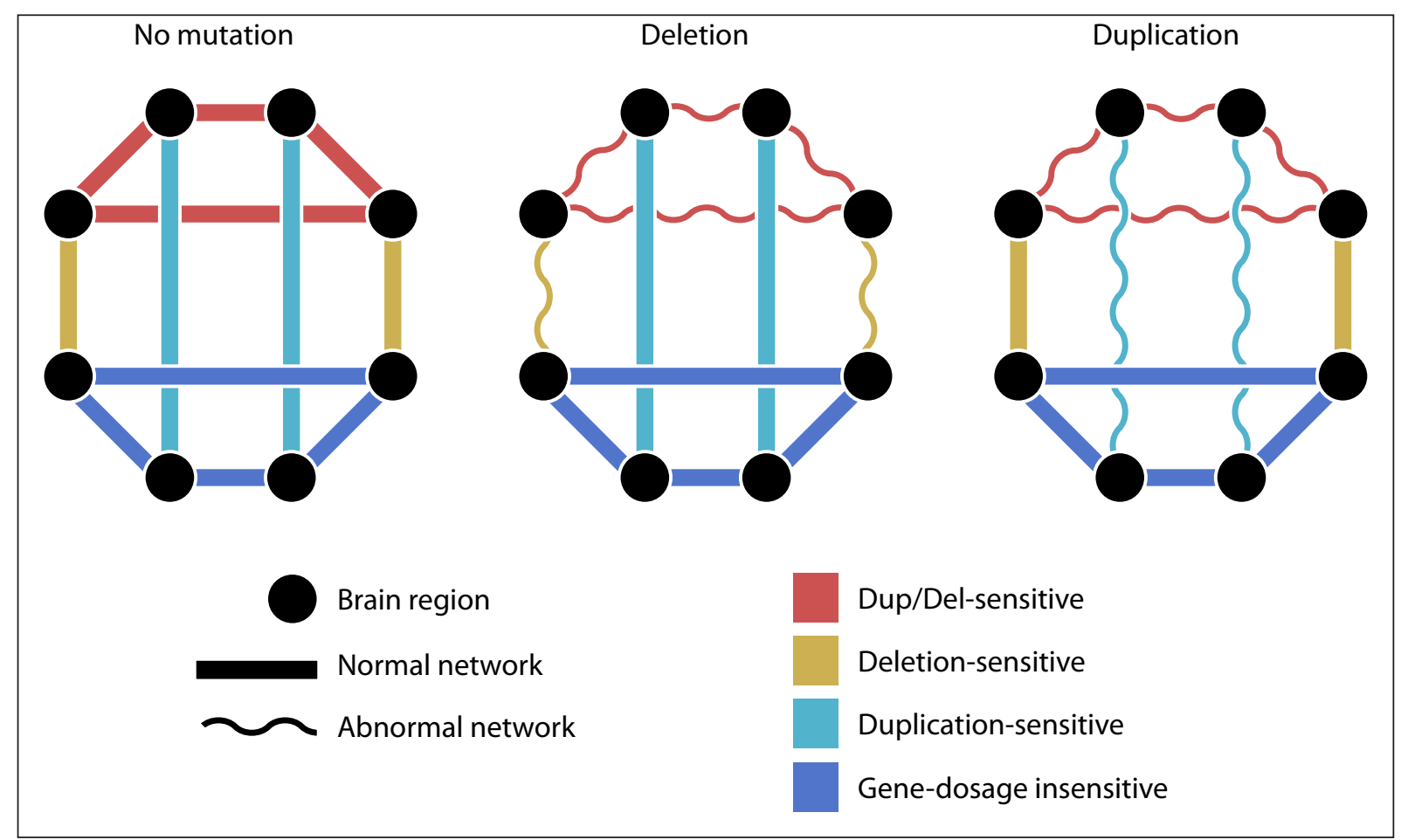

